\title{
Controlled Swimming in Confined Fluids of Magnetically Actuated Colloidal Rotors
}

\author{
Pietro Tierno, ${ }^{1,4, *}$ Ramin Golestanian, ${ }^{2}$ Ignacio Pagonabarraga, ${ }^{3,4}$ and Francesc Sagués ${ }^{1,4}$ \\ ${ }^{1}$ Departament de Química Física, Universitat de Barcelona, Martí i Franquès 1, 08028 Barcelona, Spain \\ ${ }^{2}$ Department of Physics and Astronomy, University of Sheffield, Sheffield S3 7RH, United Kingdom \\ ${ }^{3}$ Departament de Física Fonamental, Universitat de Barcelona, Martí i Franquès 1, 08028 Barcelona, Spain \\ ${ }^{4}$ Institut de Nanociència i Nanotecnologia, Universitat de Barcelona, Barcelona, Spain
}

(Received 19 June 2008; published 21 November 2008)

\begin{abstract}
We show that DNA-linked anisotropic doublets composed of paramagnetic colloidal particles can be endowed with controlled propulsion when floating above a flat plate and subjected to a magnetic field precessing around an axis parallel to the plate. The propulsion mechanism for this artificial swimmer does not involve deformations, and it makes use of the minimal two degrees of freedom needed to propel it at low Reynolds numbers. We combine experimental observations with a theoretical analysis that fully characterizes the propulsion velocity in terms of the strength and frequency of the actuating magnetic field.
\end{abstract}

Microscopic objects immersed in a fluid, whether living microorganisms [1,2], or inanimate micro-robots [3], must accommodate their motion to effectively high viscous environments. Under these conditions, inertia is negligible, and the resulting hydrodynamic laws lead to fluid flows which are time reversible [4]. This implies that swimming by reciprocal motion, i.e., based purely on symmetric backward and forward body distortions, which is characterized by a single degree of freedom, is not possible in the micro as it is the case in the macroscale (Purcell's scallop theorem [5]). One possibility is to take advantage of the strategies developed by nature, either by using the biological systems directly [6-8], or by synthesizing artificial prototypes $[9,10]$. Alternatively, self-propulsion has been achieved at microscale using nonequilibrium interfacial and phoretic effects [11-14].

A significant technological advance can be expected if we develop simpler designs for such mechanical swimmers that avoid the complexities of biological mechanisms and use very few degrees of freedom. Recent theoretical studies have shown the feasibility of such designs [15-20], but an experimental realization of such minimal low Reynolds number swimmers at the microscale is still lacking. Here, we report an experimental realization of a minimal actuated swimmer at the microscale, which makes use of the minimal two degrees of freedom required for its propulsion. Our system constitutes a new class of artificial actuated swimmers, as it does not involve body deformations; instead, its propulsion mechanism is assisted by a boundary. In particular, we show that DNA-linked assemblies of paramagnetic colloidal particles dispersed in water and floating above a flat plate can be propelled in a controlled way when subjected to a horizontal precessing magnetic field. Symmetry breaking in proximity of a boundary has been observed for bacteria (E. Coli) performing circular trajectories when swimming close to a solid surface [21]. While bacteria use rotating helical flagella to propel in viscous fluids, we here show that friction asymmetry caused by the wall is enough to achieve motion at the microscale. The proposed mechanism is based on the rectification of the cyclic rotation of the microrotors due to their asymmetric dissipation close to and far from the plate. Their motion can be understood based on the concept of a microscopic unicycle, which moves by rolling close to a solid substrate.

The anisotropic colloidal doublets are prepared by mixing monodisperse samples of streptavidin coated paramagnetic particles of two different diameters, $2 a=2.8 \mu \mathrm{m}$, $2 b=1.0 \mu \mathrm{m}$ (Dynabeads), pair-linked by means of two complementary DNA strands (25 bp, $8 \mathrm{~nm}$ long), see [22] for details. The small length of the DNA bridge ensures a strong and stiff linkage in the doublet and makes the doublet rotate as a solid body. When subjected to an external magnetic field $\boldsymbol{H}$, doublets acquire a resulting dipole moment $\boldsymbol{m}=\chi V_{d}|H| \boldsymbol{n}_{d}$ which tries to align with the field. Here, $\boldsymbol{n}_{d}$ is a unit vector along the direction of the induced dipole, $V_{d}$ denotes the doublet volume $\left(\sim 12 \mu \mathrm{m}^{3}\right)$, and $\chi$ stands for the effective magnetic susceptibility $(\sim 0.17)$. The doublets, dispersed in Millipore water at a concentration of $\sim 10^{6}$ particles $/ \mathrm{ml}$, are deposited above a clean glass plate and sediment due to gravity. Electrostatic repulsive interactions between the ionic double layer around the doublets and the negative charged substrate keep the particles floating at a distance $h$ above the plate. The external magnetic field is provided by three coils perpendicular to each other.

The applied external field precesses around a $y$-axis, parallel to the glass plate [Fig. 1(a)], with a frequency $\Omega$, $\boldsymbol{H}(t)=\left(H_{1} \sin \Omega t, H_{0}, H_{1} \cos \Omega t\right)$. A paramagnetic doublet tries to follow the field and the resulting motion is a gyroscope like precession around the $y$ axis, with the big particle practically at rest and the small one periodically tracking circular paths in the $(x, z)$ plane. As a consequence of the imposed precession, the doublet gains a net translational motion along the $x$ direction, as illustrated by the two microscopic images in Figs. 1(b) and 1(c) (see Video 1 in 


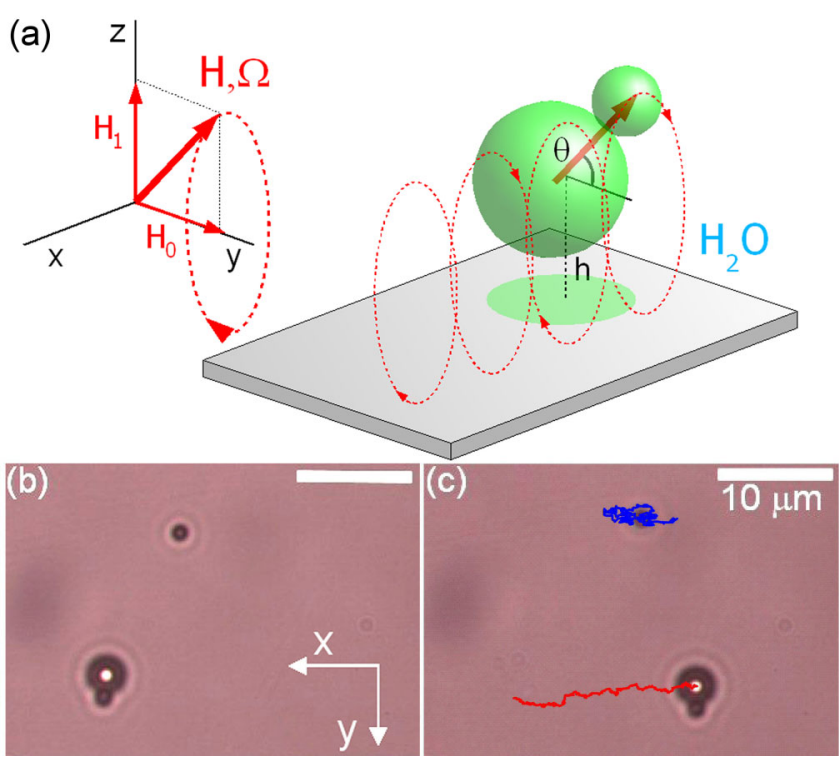

FIG. 1 (color online). (a) Schematic of a paramagnetic doublet floating above a glass plate and subjected to an external magnetic field $\boldsymbol{H}$ precessing around the $y$ axis with frequency $\Omega$. (b) and (c): microscope images showing the motion of a doublet and of an individual particle subjected to a field with $H_{0}=1223 \mathrm{~A} / \mathrm{m}$, $H_{1}=4533 \mathrm{~A} / \mathrm{m}, \Omega=62.8 \mathrm{~s}^{-1}$ at $t=0 \mathrm{~s} \mathrm{(b)} \mathrm{and} 8.9 \mathrm{~s}$ later (c).

[22]). Under the conditions of this experiment, a doublet travels a distance of $\sim 15 \mu \mathrm{m}$ in a time lapse of $8.9 \mathrm{~s}$ [see Figs. 1(c) and 2(a)]. We show in the same image the wandering motion of a single paramagnetic particle which evolves close to the tracked doublet. Its erratic motion renders an averaged displacement a hundred times smaller. Both trajectories can be characterized in terms of the mean square displacement $C(\tau)=\left\langle[\boldsymbol{r}(t+\tau)-\boldsymbol{r}(t)]^{2}\right\rangle_{t} \sim \tau^{\alpha}$, with $\boldsymbol{r}(t)$ being the particle position at time $t$. The exponent $\alpha$ characterizes the single particle motion as being diffusive for $\alpha=1$. This is clearly different from the ballistic motion, $\alpha=2$ of the doublet [see Fig. 2(b)], which remarkably lasts all over the recording time free of residual diffusion. This simultaneous observation also confirms that the doublet displacement is not an artifact attributed to some heterogeneity of the applied field, since otherwise similar propulsions for both micro-objects would have been registered. We characterize the motion of the doublet in terms of its velocity as a function of the frequency and relative strength (precession ratio) of the applied magnetic field. Fixing $H_{0} / H_{1}$ [Fig. 2(c)], the velocity of the doublet increases linearly up to a maximum value of $3.2 \mu \mathrm{m} / \mathrm{s}$ for $\Omega=70 \mathrm{~s}^{-1}$, and further decreases down to a value of $1.5 \mu \mathrm{m} / \mathrm{s}$ for $\Omega=120 \mathrm{~s}^{-1}$ [23]. Such trend in terms of the field frequency was confirmed at all the ratios $H_{0} / H_{1}$ that were investigated. By choosing appropriately the precession frequency, we can observe two distinct dependences of the doublet velocity on the precession ratio. A nearly constant velocity is observed in the low frequency regime, whereas a nonmonotonic variation is characteristic of high frequencies [two representative values of $\Omega$ are
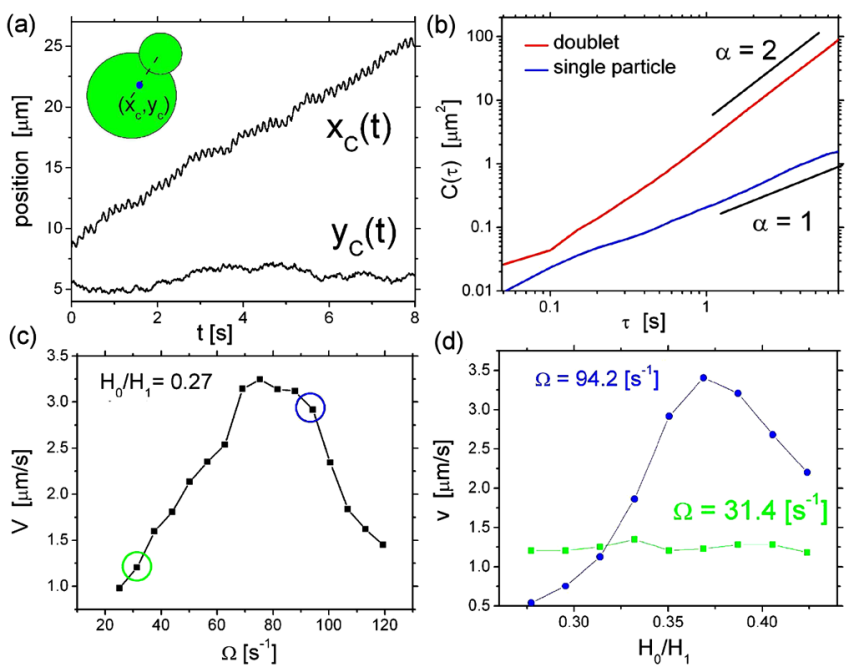

FIG. 2 (color online). (a) Reconstruction by fast video recording (125 fps) of the center-of-mass position versus time. (b) Mean square displacement versus time, for an individual particle and for a doublet distinguish diffusive $(\alpha=1)$ from directed $(\alpha=2)$ motion. (c) Doublet velocity as a function of frequency for a ratio $H_{0} / H_{1}=0.27$. (d) Doublet velocity versus field ratio $H_{0} / H_{1}$ for two frequencies, $31.4 \mathrm{~s}^{-1}$ and $94.2 \mathrm{~s}^{-1}$.

chosen in Fig. 2(d)]. To maximize the propeller efficiency, we have carried out our measurements in a field/frequency regime where the doublets synchronously rotate with the precessing field.

Reciprocal motion in viscous fluids impedes directed propulsion of either miniaturized objects or living microorganisms. As discussed in the celebrated paper by Purcell [5], a simple "scallop-like" micro-object with a single hinge cannot swim by periodically opening and closing its arms. The presence of the plate in our system is a key ingredient for breaking the time reversible symmetry of the motion. If the doublet were uniformly surrounded by a homogenous fluid, rather than floating near a plate, its cyclic motion would never render a net translational displacement. To understand the principle behind its motion, we thus have to consider the effect of the plate. Figures 3(a)-3(d), depict one such rotation cycle. In the first and third snapshots, the center of mass of the doublet is displaced dominantly along the $x$ direction, first backwards and then forwards, opposite to its tangential, i.e., horizontal, direction of motion. The clue, however, is that viscous friction due to the proximity of the plate is higher in the phase of rotation when the smaller particle in the doublet is closer to the plate, Fig. 3(c), than when it is farther away from it, Fig. 3(a). The added contribution is an uncompensated lateral translation of the small anisotropic object. Conversely, the small $z$-displacements of the center of mass are balanced between the phases of vertical direction of motion, Figs. 3(b) and 3(d), and result in no net normal translation over a cycle. In fact, periodic oscillations in the vertical location of each particle in the doublet can be experimentally observed by fast video recording, as shown 

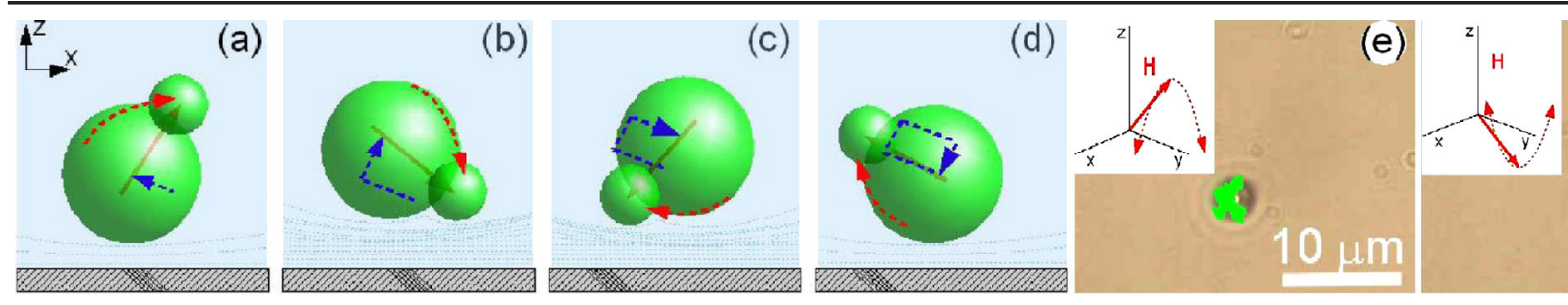

FIG. 3 (color online). (a)-(d) Schematics showing a cycle of a paramagnetic doublet subjected to a precessing magnetic field. (e), (f) Microscope images showing the position of a doublet when subjected to a magnetic field oscillating only in the plane $z>0$ (e) or $z<0$ (f). Superimposed is the particle trajectory tracked for a period of $8 \mathrm{~s}$.

in Fig. 1 in [22]. Displacements along and perpendicular to the plate are not equivalent and thus provide the two independent degrees of freedom required by Purcell's theorem to break time reciprocity. Consequently, the cycle is not invariant under time reversal due to asymmetry in dissipation far from and close to the plate; repeating the cycle continuously yields a net propulsion of the object. To confirm our reasoning, we perform a complementary experiment with a modulation $\boldsymbol{H}(t)=$ $\left(H_{1} \sin \Omega t, H_{0}, H_{1} \cos 2 \Omega t\right)$, see Figs. 3(e) and 3(f). This choice permits to oscillate the small particle back and forth alone, either above or below (depending on the sign of the ratio $H_{0} / H_{1}$ ) the equatorial line of the doublet. As expected, no direct motion of the composite particle is observed in this case, but only some marginal trembling (see Videos 2 and 3 in [22]). The same behavior will happen for any trajectory which cannot be described as a closed cycle.

We parameterized the doublet in terms of the coordinates of the centers of the two spheres $\boldsymbol{x}_{a}$ and $\boldsymbol{x}_{b}$, and the unit director $\boldsymbol{n}=\left(\boldsymbol{x}_{b}-\boldsymbol{x}_{a}\right) /(a+b)$ for fixed composite length. The velocity of each particle can be expressed in terms of the forces acting on both of them, namely, $\boldsymbol{v}_{a}=$ $\boldsymbol{G}_{a a} \boldsymbol{F}_{a}+\boldsymbol{G}_{a b} \boldsymbol{F}_{b}$, and $\boldsymbol{v}_{b}=\boldsymbol{G}_{b b} \boldsymbol{F}_{b}+\boldsymbol{G}_{b a} \boldsymbol{F}_{a}$, where $\boldsymbol{G}_{a b}$ denotes the hydrodynamic mobility matrix, which determines the velocity of particle $a$ generated by the force acting on particle $b$ [24]. The doublet motion is referred to its center-of-mass velocity $\boldsymbol{V}=d \boldsymbol{R} / d t$, with $\boldsymbol{R}$ simply expressed in terms of the mass weighted coordinates $\boldsymbol{x}_{a(b)}$ (equal density is assumed for both particles). The absence of a net force acting on the doublet implies $\boldsymbol{F}_{b}=-\boldsymbol{F}_{a}=$ $\boldsymbol{F}$, in terms of which we write formally the couple of dynamical equations for the doublet, $\boldsymbol{V}=\boldsymbol{N F}$ and $d \boldsymbol{n} / d t=\boldsymbol{M F} /(a+b)$. Here, $\boldsymbol{M}$ and $\boldsymbol{N}$ denote matrices whose elements are linear combinations of the components of the hydrodynamic mobility matrices $\left(\boldsymbol{G}_{a b}\right)$. Combining the formal expressions above, one obtains a relation between the center-of-mass velocity of the object and the precessing velocity of its director, as $\boldsymbol{V}=(a+$ b) $N \boldsymbol{M}^{-1} d \boldsymbol{n} / d t$. At the lowest order, one can neglect the hydrodynamic interactions between the particles and invert the matrix $\boldsymbol{M}$ to derive an explicit expression for the lateral and normal components of the velocity: $\boldsymbol{V}(t)=$ $\beta \frac{a^{2} \gamma\left(\gamma^{2}-\gamma+1\right)}{2 h\left(1+\gamma^{3}\right)}\left[\frac{1}{1-\Lambda_{-} n_{z}(t)}-\frac{\gamma}{1+\Lambda_{+} n_{z}(t)}\right] \frac{d n}{d t}$ with $\beta=9 / 8(9 / 4)$ for parallel (perpendicular) displacements with respect to the bounding plane, $\gamma \equiv b / a(<1)$ accounts for the size difference in the two particles of the doublet, and $\Lambda_{+} \equiv$ $a(1+\gamma) /\left[h\left(1+\gamma^{ \pm 3}\right)\right][24]$. The precession velocity of the doublet director is imposed by the applied magnetic field. The latter exerts a torque on the doublet dipole $\boldsymbol{n}_{d}$, directed along the axis $\boldsymbol{n}$, and with the same precessing angle of $\boldsymbol{H}$. Thus, $\boldsymbol{T}_{m}=\mu_{0} \boldsymbol{m} \times \boldsymbol{H}=\left(\mu_{0} V_{d} \chi|H|\right) \boldsymbol{n} \times \boldsymbol{H}$, which fixes the angular velocity of the director, $\boldsymbol{\omega}=$ $\left(1 / \zeta_{r}\right) \boldsymbol{T}_{m}$, where $\mu_{0}$ is the magnetic susceptibility of the medium and $\zeta_{r}$ stands for a doublet effective rotational friction coefficient. In our model, we consider $\zeta_{r}$ as constant since the rotational drag of the large particle dominates and the vertical displacement of the center of mass $h$ of the doublet can be neglected. Finally, we employ the kinematic equation of motion, $d \boldsymbol{n} / d t=\boldsymbol{\omega} \times \boldsymbol{n}$, which for $\boldsymbol{n}(t)=(\sin \theta \sin (\Omega t-\phi), \cos \theta, \sin \theta \cos (\Omega t-\phi))$,

where $\theta$ describes the precession angle of $\boldsymbol{n}$, leads to an expression for $\sin ^{2} \theta$ in terms of the characteristic frequency $\Omega_{B} \equiv \mu_{0} m H_{0} / \zeta_{r}$ [25]. The angle $\phi$ is the phase lag of the director with respect to the magnetic field and is found as $\tan \phi=\left(\Omega / \Omega_{B}\right) \cos \theta$. Averaging over a cycle, it turns out that $\left\langle V_{y}\right\rangle=\left\langle V_{z}\right\rangle=0$ while,

$$
\frac{\left\langle V_{x}\right\rangle}{V_{0}}=\frac{\Omega}{\Omega_{B}}\left(-\gamma-\gamma^{-3}+\frac{\gamma}{\sqrt{1-\Lambda_{+}^{2} \sin ^{2} \theta}}+\frac{\gamma^{-3}}{\sqrt{1-\Lambda_{-}^{2} \sin ^{2} \theta}}\right)
$$

where $V_{0}=\frac{9}{16} \frac{a \gamma\left(\gamma^{2}-\gamma+1\right)}{1+\gamma} \Omega_{B}$ is a characteristic velocity scale. We notice that this is a general expression valid also for $\gamma=1$ (equal size spheres).

To test the model, we perform a series of experiments varying the precession ratio and the frequency. Figure 4 compares the experimental result with the theoretical model, leaving $h$ and $\zeta_{r}$ as adjustable parameters. The comparison shows that, despite the simplifications in the model, we have quantitative agreement over all the explored range of parameter values. Increasing $\Omega$ at small frequencies linearly increases the rate of rectification of the cyclic rotation, while the variation of the angle $\theta$ is small. Conversely, at large frequencies, the angle $\theta$ approaches zero and the small particle rotates further away from the plane, this lowering the efficiency in rectification per cycle. Instead, decreasing the ratio $H_{0} / H_{1}$ increases the initial value of $\theta$ and thus shifts to higher frequencies the peak of 


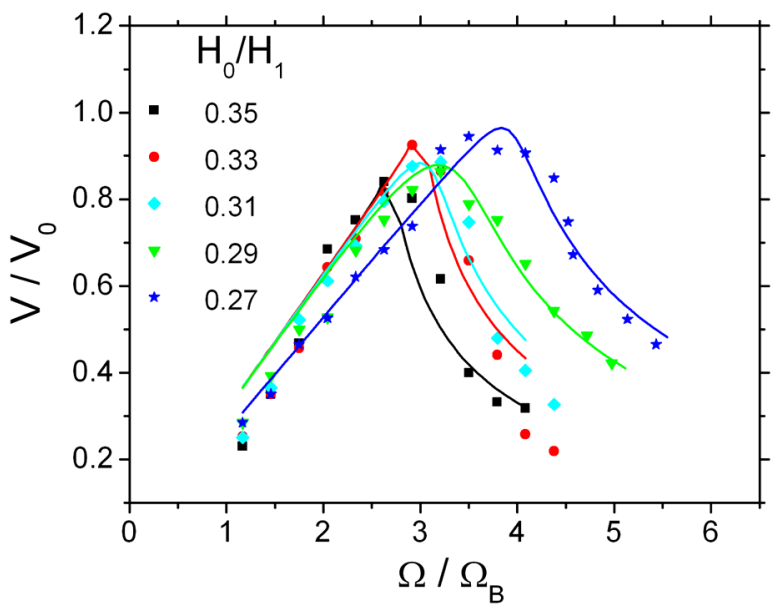

FIG. 4 (color online). Scaled doublet velocity as a function of frequency for different precession ratios $H_{0} / H_{1}$. Points denote experimental data while continuous lines are fits following the theoretical model introduced in the text.

the doublet velocity. The result of the theoretical fitting allows us to extract an average distance from the center of mass to the plate as $h=2.1 \pm 0.1 \mu \mathrm{m}$ and a value of the rotational friction coefficient $\zeta_{r}=8.6 \times 10^{-19} \mathrm{~N} \cdot \mathrm{s} \cdot \mathrm{m}$.

The method of propulsion will hold for any externally driven object rotating close to a solid surface since it will experience a rotational-translational coupling [26,27]. We choose to use an elongated doublet since it represents the simplest microscale object which can be built and rotate starting from paramagnetic particles. Other assemblies like ferromagnetic spherical particles, chains of paramagnetic particles, and more complex architectures will be equally propelled.

In summary, we have experimentally demonstrated and theoretically described a principle of directed propulsion based on the rectification of the solid rotation of a magnetically driven colloidal doublet immersed in a viscous dominated confined fluid. This example makes use of the minimal two degrees of freedom for propulsion at low Reynolds number, and it does not involve body deformations. The artificial swimmer is actuated in a nonintrusive way through magnetic fields, can be readily scaled down to nanoscale, and can be efficiently steered. It takes advantage of bounding planes, which makes it particularly suited to operating in microfluidic devices, unlike other swimmers whose mobility will be hindered by channel boundaries either due to friction forces or by depleting the chemicals needed to power them. Although our realization is based on anisotropic composites of microparticles, many other elongated magnetic micro or nano object could be equally actuated such as, for example, ferromag- netic nanorods [28], magnetotactic bacteria [29], or ferrofluid doped microphages [30].

P. T. was supported by the program "Beatriu de Pinós" BP-B100167. F. S. and P. T. acknowledge financial support by MEC (Project FIS2006-03525) and DURSI (2005SGR00653), R.G. acknowledges support from the EPSRC. I.P. acknowledges financial support from MEC (Project FIS2005-01299).

*ptierno@ub.edu

[1] G. I. Taylor, Proc. R. Soc. A 209, 447 (1951).

[2] H. C. Berg and R. A. Anderson, Nature (London) 245, 380 (1973).

[3] B. Behkam and M. Sitti, Appl. Phys. Lett. 90, 023902 (2007).

[4] J. Happel and H. Brenner, Low Reynolds Number Hydrodynamics (Noordhoff, Leiden, 1973).

[5] E. M. Purcell, Am. J. Phys. 45, 3 (1977).

[6] H. C. Berg, Annu. Rev. Biochem. 72, 19 (2003).

[7] S. L. Tamn, J. Cell Biol. 55, 250 (1972).

[8] K. M. Ehlers et al., Proc. Natl. Acad. Sci. U.S.A. 93, 8340 (1996).

[9] H. Morimoto et al., Phys. Rev. E 78, 021403 (2008).

[10] R. Dreyfus et al., Nature (London) 437, 862 (2005).

[11] W. F. Paxton et al., J. Am. Chem. Soc. 126, 13424 (2004).

[12] S. Thakur et al., Phys. Rev. Lett. 97, 115701 (2006).

[13] J. R. Howse et al., Phys. Rev. Lett. 99, 048102 (2007).

[14] S. T. Chang et al., Nature Mater. 6, 235 (2007).

[15] H. A. Stone and A. D. T. Samuel, Phys. Rev. Lett. 77, 4102 (1996).

[16] A. Najafi and R. Golestanian, Phys. Rev. E 69, 062901 (2004).

[17] J. E. Avron, O. Gat, and O. Kenneth, Phys. Rev. Lett. 93, 186001 (2004).

[18] T. S. Yu, E. Lauga, and E. Hosoi, Phys. Fluids 18, 091701 (2006).

[19] C. M. Pooley et al., Phys. Rev. Lett. 99, 228103 (2007).

[20] F. Y. Ogrin et al., Phys. Rev. Lett. 100, 218102 (2008).

[21] E. Lauga et al., Biophys. J. 90, 400 (2005).

[22] See EPAPS Document No. E-PRLTAO-101-007847 for supplementary videos. For more information on EPAPS, see http://www.aip.org/pubservs/epaps.html.

[23] With this value, we estimate the Reynolds number in our system: $R e=\mathrm{aV}_{0} / \nu \sim 10^{-8}$.

[24] P. Tierno, R. Golestanian, I. Pagonabarraga, and F. Sagués (to be published).

[25] We find: $\sin ^{2} \theta=1-\frac{1}{2}\left(\Omega_{B} / \Omega\right)^{2}\left[\left(\Omega / \Omega_{B}\right)^{2}-\left(H_{1} / H_{0}\right)^{2}-\right.$ $\left.1+\sqrt{\left[1+\left(H_{1} / H_{0}\right)^{2}-\left(\Omega / \Omega_{B}\right)^{2}\right]^{2}+4\left(\Omega / \Omega_{B}\right)^{2}}\right]$.

[26] A. J. Goldman, R. G. Cox, and H. Brenner, Chem. Eng. Sci. 22, 637 (1967).

[27] A. M. J. Davis, M. T. Kezirian, and H. Brenner, J. Colloid Interface Sci. 165, 129 (1994).

[28] P. Dhar et al., Nano Lett. 7, 1010 (2007).

[29] E. Wajnberg et al., Biophys. J. 50, 451 (1986).

[30] P. Dhar et al., J. Phys. Chem. B 111, 13097 (2007). 